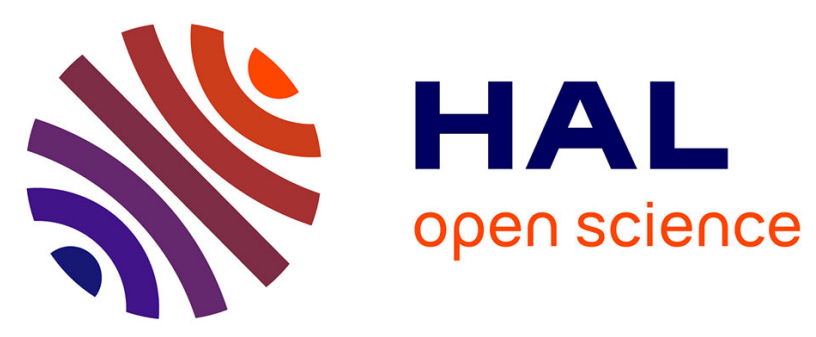

\title{
Functional evidence implicating FOXL2 in non syndromic premature ovarian failure and in the regulation of the transcription factor OSR2.
}

Paul Laissue, Besma Lakhal, Bérénice A Benayoun, Aurélie Dipietromaria, Rim Braham, Hatem Elghezal, Pascal Philibert, Ali Saâd, Charles Sultan, Marc Fellous, et al.

\section{To cite this version:}

Paul Laissue, Besma Lakhal, Bérénice A Benayoun, Aurélie Dipietromaria, Rim Braham, et al.. Functional evidence implicating FOXL2 in non syndromic premature ovarian failure and in the regulation of the transcription factor OSR2.. Journal of Medical Genetics, 2009, 46 (7), pp.455. 10.1136/jmg.2008.065086 . hal-00552682

\section{HAL Id: hal-00552682 https://hal.science/hal-00552682}

Submitted on 6 Jan 2011

HAL is a multi-disciplinary open access archive for the deposit and dissemination of scientific research documents, whether they are published or not. The documents may come from teaching and research institutions in France or abroad, or from public or private research centers.
L'archive ouverte pluridisciplinaire HAL, est destinée au dépôt et à la diffusion de documents scientifiques de niveau recherche, publiés ou non, émanant des établissements d'enseignement et de recherche français ou étrangers, des laboratoires publics ou privés. 


\section{Functional evidence implicating FOXL2 in non syndromic premature ovarian failure and in the regulation of the transcription factor OSR2.}

Paul Laissue $^{1,2 *}$, Besma Lakhal ${ }^{3 *}$, Bérénice Benayoun ${ }^{1,2,5}$, Aurélie Dipietromaria ${ }^{1,2}$, Rim Braham $^{4}$, Hatem Elghezal ${ }^{3}$, Pascal Philibert ${ }^{6,7,8}$, Ali Saâd ${ }^{3}$, Charles Sultan ${ }^{6,7}$, Marc Fellous ${ }^{1,2}$, and Reiner A. Veitia $^{1,2,8}$

${ }^{1}$ Institut Cochin, Université Paris Descartes, CNRS (UMR 8104), Paris, France. ${ }^{2}$ Inserm, U567, Paris, France. ${ }^{3}$ Department of Cytogenetics and Reproductive Biology, Farhat Hached University Teaching Hospital, Sousse, Tunisia. ${ }^{4}$ Department of Endocrinology, Farhat Hached University Teaching Hospital, Sousse, Tunisia. ${ }^{5}$ École Normale Supérieure, 75005 Paris, France. ${ }^{6}$ Unité d'Endocrinologie-Gynécologie Pédiatrique, Service de Pédiatrie, Hôpital Arnaud-de-Villeneuve and Service d'Hormonologie du Développement et de la Reproduction, Hôpital Lapeyronie, Centre Hospitalier Universitaire Montpellier, Montpellier, France. ${ }^{7}$ Institut de Génétique Humaine, Centre Nationale de la Recherche Scientifique UPR1142, Montpellier, France. ${ }^{8}$ Université Paris-Diderot, Paris VII, 75013 Paris, France.

*These authors contributed equally to this work and are listed alphabetically.

Corresponding author: R. A. Veitia (reiner.veitia@inserm.fr) 


\section{Abstract}

Background: FOXL2 encodes a forkhead transcription factor whose mutations are responsible for the Blepharophimosis-Ptosis-Epicanthus inversus Syndrome (BPES), involving craniofacial/palpebral abnormalities often associated with premature ovarian failure (POF).

Results: We describe a FOXL2 variant (p.Gly187Asp) in a case of POF without BPES. The subcellular localization of FOXL2-G187D was normal but its transactivation capacity tested on two reporter promoters potentially relevant to the ovary was significantly lower than that of normal FOXL2. However, FOXL2-G187D was able to strongly activate a reporter construct driven by the promoter of Osr2 (odd-skipped related 2 transcription factor), which we have suggested to be a crucial target of FOXL2 in the craniofacial region. This is compatible with the absence of BPES in our patient.

Conclusions: Our data provide evidence in favor of the implication of FOXL2 variants in nonsyndromic POF and confirm the regulatory interaction between FOXL2 and OSR2 whose perturbation might contribute to the palpebral abnormalities observed in BPES patients. 


\section{Short Report}

FOXL2 is a single-exon gene encoding a forkhead transcription factor ${ }^{1}$ expressed in the ovarian somatic compartment and peri-ocular tissues ${ }^{2}$. In humans, its mutations cause the Blepharophimosis-Ptosis-Epicanthus inversus Syndrome (BPES), which involves craniofacial/palpebral abnormalities associated or not with premature ovarian failure $(\mathrm{POF})^{3}$.

Here, we study the effects of a FOXL2 variant detected in a POF case without BPES. Clinically, the 27 year-old patient of Tunisian origin, presented with secondary amenorrhea. She had a normal pubertal development and no history of diabetes, pelvic surgery, radiation or autoimmunity. Consanguinity and familial history of infertility were excluded. She had high FSH (45 IU/L; normal menopausal range: 25-160 IU/L) and LH (35 IU/L; normal menopausal range: 11.3-40 IU/L) levels and low E2 (20 pg/mL; normal menopausal range $<55 \mathrm{pg} / \mathrm{mL})$. Cytogenetic analysis showed a normal 46,XX karyotype. Pelvic and ultrasound examination showed normal uterus and ovaries with several small follicles. The patient had none of the typical features of BPES (A signed informed consent is available from the JMG website).

Direct sequencing of the FOXL2 coding region (performed as previously described ${ }^{4}$ ) revealed the presence of the heterozygous variant c.560G $>$ A, inherited from her father, leading to the amino acid substitution p.Gly187Asp (FOXL2-G187D) in a highly conserved segment, C-terminal to the forkhead DNA-binding domain (Figure 1). This variant was absent in 110 control chromosomes. To assess the potential deleterious effect of the amino acid change we used the SIFT software (http://blocks.fhcrc.org/sift/SIFT.html). SIFT uses protein sequence conservation data to calculate the probability of a substitution of being deleterious. Scores lower than 0.05 suggest a potential 
pathogenicity. The score of p.Gly187Asp, obtained using the alignment shown in Figure 1, was 0. The substitution p.Gly187Asp implies an important change in polarity. Glycine is a small neutral amino acid whereas aspartate is acidic and negatively charged at $\mathrm{pH} 7$. Interestingly, Tyr186 is predicted to be phosphorylatable (http://www.cbs.dtu.dk/services/NetPhosK/). Therefore, we hypothesize that the negative charge of the side chain of aspartate might interfere with the posttranslational modification process or mimic the modification itself, perturbing ovarian-specific protein interactions and/or regulations.

To assess subcellular localization and transcriptional activity of FOXL2-G187D, we performed transfection experiments and luciferase reporter assays as previously described ${ }^{5,6}$. The FOXL2G187D variant fused to the green fluorescent protein displayed a typical nuclear staining, indistinguishable from that of normal FOXL2. From a functional perspective, FOXL2-G187D was found to activate the promoter of FOXL2 itself and the FOXL2-specific artificial promoter 2xFLRE-luc (containing 2 FOXL2 response elements upstream of a minimal CMV promoter) ${ }^{6}$, though significantly less strongly than the normal protein. The p.Ile84Ser mutant, responsible for a type I BPES ${ }^{7}$, was used as a negative control and was, as expected, unable to transactivate our luciferase reporters. We have recently suggested that a positive feedback loop of FOXL2 (which activates its own promoter) might be important in response to oxidative stress in the ovary ${ }^{8}$. Thus, we hypothesize that a lowered transactivation capacity of FOXL2-G187D on ovarian targets might explain the ovarian phenotype in our patient (Figure 2 A, B). However, the effects of this variant is expected to be promoter-dependent, as previously shown for other mutants ${ }^{5,6}$, and as shown below for the third reporter promoter that we tested. 
We have previously suggested that, in the craniofacial region, the encoding odd-skipped related 2 transcription factor (OSR2) should be a crucial target of FOXL2 ${ }^{9}$. Osr2 is, as Foxl2, highly expressed in the murine periocular mesenchyma ${ }^{10,11}$. Moreover, as described for Foxl2 $2^{-/-}$mice, $O s r 2^{-/-}$mutant neonates exhibit open eyelids with similar abnormalities ${ }^{10,11}$. Unfortunately, no data concerning the $O s r 2^{-/-}$ovary are available yet ${ }^{10,12}$. In agreement with our hypothesis and with the absence of an eyelid phenotype in our patient (and in her mutation-carrier father), we found that FOXL2-G187D was able to strongly activate a reporter construct driven by the Osr2 promoter (pOSR2) described by Kawai et al $2005^{12}$. Coherently, the FOXL2-I84S mutant found in a BPES I patient failed to transactivate pOsr2 (Figure $2 \mathrm{C}$ ). Since no second BPES locus has been reported thus far, it is unlikely that mutations in OSR2 itself might contribute directly to BPES. However, regulatory mutations perturbing the interaction FOXL2-pOSR2 are worth being screened in BPES patients with normal FOXL2.

The fact that FOXL2-G187D may be either normal or hypomorph suggests that it can behave as a susceptibility variant whose activity might be shifted up or down by modifier genes, leading to normal ovarian function or POF, respectively. Given our functional evidence, it would be interesting to formally assess the association between p.Gly187Asp and POF.

A previous mutation screening has detected a $30 \mathrm{bp}$ deletion removing 10 out of 14 alanines of the FOXL2 polyAlanine tract (i.e. FOXL2-Ala4) and the substitution p.Tyr258Asn in two POF patients $^{13,14}$. Interestingly, neither mutation was identified in 200 control chromosomes. However, the fact that the p.Tyr258Asn variant was maternally inherited and that the patient carrying the FOXL2-Ala4 variant spontaneously conceived and delivered two babies did not 
allow a formal causal implication of these alterations in absence of functional assays at the time of these studies. Both variants might display incomplete penetrance or lead, at least, to partial fertility recovery after appropriate treatments. Thus, it would be interesting to assess the functional impact for both alterations.

Taken together, our results provide functional evidence in favor of the implication of FOXL2 variants in non-syndromic POF and confirm the regulatory interaction between FOXL2 and OSR2, whose perturbation might contribute to the palpebral phenotype in BPES. In our opinion, a more systematic genetic screening of FOXL2 mutations is of interest in non-syndromic POF, to improve genetic counseling and to better understand the molecular etiology of this frequent pathology.

Acknowledgments. We wish to thank S. Kawai for sharing with us the reporter plasmid driven by the pOsr2 and two anonymous referees for their constructive comments. Our work is supported by the INSERM, the CNRS, the Fondation pour la Recherche Médicale (FRM) and the Institut Universitaire de France (IUF).

Licence for Publication. "The Corresponding Author has the right to grant on behalf of all authors and does grant on behalf of all authors, an exclusive licence (or non exclusive for government employees) on a worldwide basis to the BMJ Publishing Group Ltd to permit this article (if accepted) to be published in Journal of Medical Genetics and any other BMJPGL products and sublicences such use and exploit all subsidiary rights, as set out in our licence (http://JMG.bmj.com/misc/ifora/licenceform.shtml)." 


\section{References.}

1. Crisponi L, Deiana M, Loi A, Chiappe F, Uda M, Amati P, Bisceglia L, Zelante L, Nagaraja R, Porcu S, Ristaldi MS, Marzella R, Rocchi M, Nicolino M, Lienhardt-Roussie A, Nivelon A, Verloes A, Schlessinger D, Gasparini P, Bonneau D, Cao A, Pilia G. The putative forkhead transcription factor FOXL2 is mutated in blepharophimosis/ptosis/epicanthus inversus syndrome. Nat Genet 2001; 27:159-166.

2. Cocquet J, Pailhoux E, Jaubert F, Servel N, Xia X, Pannetier M, De Baere E, Messiaen L, Cotinot C, Fellous M, Veitia RA. Evolution and expression of FOXL2. J Med Genet 2002; 39:916-921.

3. Zlotogora J, Sagi M, Cohen T. The blepharophimosis, ptosis, and epicanthus inversus syndrome : delineation of two types. Am J Hum Genet 1983; 35:1020-1027.

4. De Baere E, Lemercier B, Christin-Maitre S, Durval D, Messiaen L, Fellous M, Veitia R. FOXL2 mutation screening in a large panel of POF patients and XX males. J Med Genet 2002; 39:e43.

5. Moumne L, Dipietromaria A, Batista F, Kocer A, Fellous M, Pailhoux E, Veitia RA. Differential aggregation and functional impairment induced by polyalanine expansions in FOXL2, a transcription factor involved in cranio-facial and ovarian development. Hum Mol Genet 2008; 17:1010-1019.

6. Benayoun BA, Caburet S, Dipietromaria A, Bailly-Bechet M, Fellous M, Vaiman D, Veitia RA. The identification and characterization of a FOXL2 response element provides insights into the pathogenesis of mutant alleles. Hum Mol Genet 2008; 17:3118-3127.

7. Dollfus H, Stoetzel C, Riehm S, Lahlou Boukoffa W, Bediard Boulaneb F, Quillet R, Abu-Eid M, Speeg-Schatz C, Francfort JJ, Flament J, Veillon F, Perrin-Schmitt F. Sporadic and 
familial blepharophimosis -ptosis-epicanthus inversus syndrome: FOXL2 mutation screen and MRI study of the superior levator eyelid muscle. Clin Genet 2003; 63:117-120.

8. Benayoun BA, Batista F, Auer J, Dipietromaria A, L'hôte D, De Baere E, Veitia RA. Positive and negative feedback regulates the transcription factor FOXL2 in response to cell stress: evidence for a regulatory imbalance induced by disease-causing mutations. Hum Mol Genet 2009; 18:632-644.

9. Batista F, Vaiman D, Dausset J, Fellous M, Veitia RA. Potential targets of FOXL2, a transcription factor involved in craniofacial and follicular development, identified by transcriptomics. Proc Natl Acad Sci U S A 2007; 104:3330-3335.

10. Lan Y, Ovitt CE, Cho ES, Maltby KM, Wang Q, Jiang R. Odd-skipped related 2 (Osr2) encodes a key intrinsic regulator of secondary palate growth and morphogenesis. Development 2004; 131:3207-3216.

11. Uda M, Ottolenghi C, Crisponi L, Garcia JE, Deiana M, Kimber W, Forabosco A, Cao A, Schlessinger D, Pilia G. Foxl2 disruption causes mouse ovarian failure by pervasive blockage of follicle development. Hum Mol Genet 2004; 13:1171-1181.

12. Kawai S, Kato T, Inaba H, Okahashi N, Amano A. Odd-skipped related 2 splicing variants show opposite transcriptional activity. Biochem Biophys Res Commun 2005; 328:306311.

13. Harris SE, Chand AL, Winship IM, Gersak K, Aittomaki K, Shelling AN. Identification of novel mutations in FOXL2 associated with premature ovarian failure. Mol Hum Reprod 2002; 8:729-733. 
14. Gersak K, Harris SE, Smale WJ, Shelling AN. A novel 30 bp deletion in the FOXL2 gene in a phenotypically normal woman with primary amenorrhoea: case report. Hum Reprod 2004; 19:2767-2770. 


\section{Figure legends.}

Figure 1: FOXL2 sequence alignment around Gly187. The alignment involves sequences from vertebrates ranging from amphibian and fishes to mammals. The relevant Gly187 residue is highlighted within a frame. The immediately previous highly conserved Tyr/Y residue is predicted to be phosphorylatable. The negative charge of aspartate in FOXL2-Gly187Asp might interfere with the post-translational modification process or mimic the modification itself.

Figure 2. Luciferase assays using three reporter promoters. For transfections, we used granulosalike KGN cells and each experiment was performed in 5 replicates. The variant p.Ile84Ser was obtained by fusion PCR as previously described ${ }^{5,6}$. A) Activity on the FOXL2-specific artificial promoter 2xFLRE-luc (containing 2 FOXL2 response elements upstream of a minimal CMV promoter $^{6}$, B) Activity on the promoter of FOXL2 itself and C) Activity on pOsr2. In all cases ANOVAs were significant $(\mathrm{p}<0.001)$. Post-hoc Tuckey HSD tests showed that the mutant p.Ile84Ser always behaved no differently than the empty control vector, while G187D had a significant activity, through lower than the wild-type/WT protein for 2xFLRE-luc and pFOLX2luc (A,B). Interestingly, in the case of pOsr2-luc (C), G187D had an activity even stronger than the WT. In all cases, p-values were at least $<0.05$. 
Homo_sapiens Pan_troglodytes Bos_taurus Capra_hircus Pteropus_vampyrus Sus_scrofa Dasypus_novemcinctus Ellobius_lutescens Oryctolagus_cuniculus Mus_musculus Epinephelus_merra Oreochromis_aureus Oryzias_luzonensis Oncorhynchus_mykiss Danio_rerio Silurus_meridionalis Xenopus_laevis Rana_rugosa Gallus_gallus scyliorhinus_canicula
MKRPFRPP PAHFQPGKGLFGAGGAAGGCGVAGAGADGYGPLAPPKYLQ MKRPFRPPPAHFQPGKGLFGAGGAAGGCGVAGAAADGYGYLAP PKYLQ MKRPFRPPPAHFQPGKGLFGAGGAAGGCGVAGAGADGYGYLAPPKYLQ MKRPFRPPPAHFQPGKGLFGAGGAAGGCGVAGAGADGYGYLAPPKYLQ MKRPFRPPPAHFQPGKGLFGAAGAAGGCGVAGAGTDGYGYLAPPKYLQ MKRPFRPPPAHFQPGKGLFGAGGAAGGCGVAGAGADGYGYLAPPKYLQ MKRPFRPPPAHFQPGKGLFAAGGAAAGCGVAGAGAD SYGYLAPPKYLQ MKRPFRPPPAHFQPGKGLFGSGGAAGGCGVAGAGADGYGYLAPPKYLQ MKRPFRPPPAHFQPGKGLFGAAGAAGGCGVAGAGADGYGYLAPPKYLQ MKRPFRPPPAHFQPGKGLFGSGGAAGGCGVPGAGADGYGYLAPPKYLQ MKRPFRPPPTHFQP - - - - - - - - - GKSLFGGDGYGYLSPPKYLQ MKRPFRPPPTHFQP - - - - - - - - GKALFGGDSYGYLSPPKYLQ MKRPFRPPPTHFQP - - - - - - - - - GKALFGGDGYGYLSPPKYLQ MKRPFRPPPTHFQP - - - - - - - - - GKSLFGGDGYGYLSPPKYLQ MKRPFRPPPTHFQP - - - - - - - - - - GKSLFGGEGYGYLSPPKYLQ MKRPFRPPTSHFQA - - - - - - - - - GKSIFGGDSYGYLSPPKYLQ MKRPFRPPPTHFQA - - - - - - - - GKSLFGSDTYGYLSPPKYLQ MKRPFRPP PTHFQA - - - - - - - - - -GKSLF SSDTYGYLSPPKYLQ MKRPFRPPPTHFQP - - - - - - - - GKSLFGPDGYGYLSPPKYLQ MKRPFRPPPTHFQHS - - - - - - - - - KAALFASDSYGYIGPPKYLQ 
